BIO Web of Conferences 6, 03006 (2016)

DOI: $10.1051 /$ bioconf/20160603006

(C) Owned by the authors, published by EDP Sciences, 2016

\title{
Green thermoelectrics: Observation and analysis of plant thermoelectric response
}

\author{
Christophe Goupil ${ }^{1}$, Henni Ouerdane ${ }^{2}$, Éric Herbert ${ }^{1, \text { a }}$, Arnold Kamsing ${ }^{1}$, Yann Apertet ${ }^{3}$, François \\ Bouteau $^{1}$, Stefano Mancuso ${ }^{4}$, Rodrigo Patino ${ }^{1,5}$ and Philippe Lecoeur ${ }^{6}$ \\ ${ }^{1}$ Laboratoire Interdisciplinaire des Energies de Demain (LIED) UMR 8236, Université Paris Diderot CNRS 4 Rue \\ Elsa Morante 75013 Paris France \\ ${ }^{2}$ Russian Quantum Center, 100 Novaya Street, Skolkovo, Moscow region 143025, Russia3 \\ 3 Lycée Jacques Prévert, 30 Route de Saint Paul, 27500 Pont-Audemer, France \\ ${ }^{4}$ Laboratorio Internazionale di Neurobiologia Vegetale - Department of Plant Soil I\& Environmental Science, \\ University of Florence, Florence, Italy \\ ${ }^{5}$ Departamento de Fisica Aplicada, Cinvestav-Unidad Mérida, AP 73 Cordemex, 97310 Mérida, Yucatan, Mexico \\ ${ }^{6}$ Institut d'Electronique Fondamentale, Université Paris-Sud, CNRS, UMR 8622, F-91405 Orsay, France
}

\begin{abstract}
Plants are sensitive to thermal and electrical effects; yet the coupling of both, known as thermoelectricity, and its quantitative measurement in vegetal systems never were reported. We recorded the thermoelectric response of bean sprouts under various thermal conditions and stress. The obtained experimental data unambiguously demonstrate that a temperature difference between the roots and the leaves of a bean sprout induces a thermoelectric voltage between these two points. Basing our analysis of the data on the force-flux formalism of linear response theory, we found that the strength of the vegetal equivalent to the thermoelectric coupling is one order of magnitude larger than that in the best thermoelectric materials. Experimental data also show the importance of the thermal stress variation rate in the plant's electrophysiological response. therefore, thermoelectric effects are sufficiently important to partake in the complex and intertwined processes of energy and matter transport within plants.
\end{abstract}

\footnotetext{
${ }^{\mathrm{a}}$ Corresponding author: eric.herbert@univ-paris-diderot.fr
} 